\title{
Alternative estimation of effective Young's Modulus for Lightweight Aggregate Concrete LWAC
}

\author{
Meriem Fakhreddine Bouali \\ Department of Civil Engineering, Faculty of Sciences \& Technology, University of Mohamad Cherif Messaadia, Souk Abras, \\ 41000, Algeria \\ m.bouali@univ-soukahras.dz.b.meriemfakhreddine@gmail.com,bttp://orcid.org/0000-0002-6986-980X
}

\author{
Abdelkader Hima \\ Department of Electrical Engineering, Faculty of Technology, University of El-Oued, 39000, Algeria \\ Abdelkader-hima@univ-eloued.d», bttp://orcid.org/0000-0002-5533-3991
}

\begin{abstract}
The prediction of effective mechanical properties of composite materials using analytical models is of significant practical interest in situations in which tests are impossible, difficult, or costly. Many experimental and numerical works are attempting to predict the elastic properties of Lightweight Aggregate Concrete (LWAC). In order to choose the optimized prediction composite model, the purpose of this paper is to appraise the effective Young's modulus of LWAC using two-phase composite models. To this effect, results of previous experimental research have used as a platform, upon which, 07 two-phase composite models were applied. The outcomes of this comparative analysis show that not all two-phase analytical models can be directly used for predicting Young's modulus of LWAC. The Maxwell, Counto1 and Hashin-Hansen models are in close concordance with the experimental Young's modulus of all LWAC used for comparison in this study (119 values). They were found more appropriate for reasonable prediction of elasticity modules of the LWAC.
\end{abstract}

KEYwORDS. Analytic model; Concrete; Young's modulus; Lightweight aggregate; Two-phase.

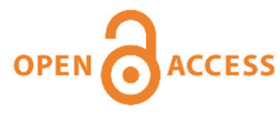

Citation: Bouali, M. F., Hima, A. Alternative estimation of effective Young's Modulus for Lightweight Aggregate Concrete LWAC, Frattura ed Integrità Strutturale, 52 (2020) 8297.

Received: 15.11 .2019

Accepted: 08.01 .2020

Published: 01.04 .2020

Copyright: (C) 2020 This is an open access article under the terms of the CC-BY 4.0, which permits unrestricted use, distribution, and reproduction in any medium, provided the original author and source are credited.

\section{INTRODUCTION}

$\mathrm{R}$ ecently, special attention has been paid to the development of Lightweight Aggregate Concrete (LWAC) [1, 2, 3] which offers many advantages as a building material, including low weight, easier construction and better resistance compared with ordinary concrete. Lightweight Aggregate Concrete (LWAC) primarily improves the thermal and sound insulation properties of buildings next to its basic applications [4]. The lightweight concrete are created by substituting the natural aggregates with the lightweight aggregates (LWA), which are classified into two fundamental 
categories: natural (like pumice, diatomite, volcanic ash, etc.) and manufactured (such as perlite, extended schist, clay, slate, sintered powdered fuel ash (PFA), etc.) [3, 5]. Beside its technical and financial interests, LWAC can be integrated into the demarche of sustainable improvement by utilizing in specific artificial aggregates which are lighter than natural aggregates [6].

The Young's modulus (elastic modulus) is a very important material property which is measured directly on concrete. Engineers need to know the value of this parameter to conduct any computer simulation of structure. Various experimental works have concerned the study of behavior of LWAC $[1,7,8,9,10,11,12]$. However from an experimental point of view, this is not always easy. Therefore when the tests are impossible, difficult, costly, or timeconsuming, the research about prediction models for the elastic modulus using properly validated composite models is of great practical interest.

The aim of the composites materials approach is to develop a model that will enable expression of average properties of the mixtures through properties and volume fractions of its constituents [11]. Diverse explicit models of the literature are utilized. Their application to the prediction of LWAC behaviors shows a wide dissimilarity between the different approaches particularly when the volume fraction of reinforcement is more than $40 \%$ and when the contrast between the phases grows [9].

For this purpose and to distinguish the most appropriate two-phase composite model for predicting LWAC's effective modulus of elasticity, the estimation of the Young's modulus of LWAC using two-phase composite models was applied. Furthermore, an efficient and accurate model is useful to reduce the cost and duration of the experimental mix design studies.

In this present work, a large bibliography data for different LWAC tested experimentally and published in the literature are used: De Larrard [7], Yang and Huang [8], and Ke Y et al. [9].

For LWAC test results investigated in this study, the volume fraction $\mathrm{V}_{\mathrm{g}}$ of the lightweight aggregate varies from $0 \%$ (the matrix) to $47.8 \%$ and the contrast of the characteristics of the phases $\mathrm{E}_{\mathrm{g}} / \mathrm{E}_{\mathrm{m}}$ (Young's modulus of lightweight aggregate and matrix) varies between $0.20 \%$ and $95 \%$ except for four types of concretes for which this ratio exceeds 1 because of a very low value of $E_{m}\left(E_{g}>E_{m}\right)[7]$.

In order to determine the models likely to yield the lowest number of errors; the results of effective Young's modulus of LWAC obtained by using 07 two-phase composite models were compared with the experimental results obtained by De Larrard [7], Yang and Huang [8] and Ke Y et al. [9] (119 values) and discussed. Therefore, prediction possibilities using composite material models in determination of modulus of elasticity were sought and some suggestions were made accordingly to a statistical study.

\section{PREDiCTION OF ELASTIC MODULUS FOR LWAC}

\section{Two-phase composite models}

$\mathrm{M}$ Ore attention has been paid to lightweight aggregate concrete. The weakest component of LWAC is not the cement matrix or the interfacial transition zone (ITZ) but the aggregates. Therefore, the research about prediction model for LWAC's Young modulus is valuable for the concrete application [6]. Lo and Cui [13] illustrate that the "Wall effect" does not exist on the surface of expanded clay aggregates in lightweight concrete by SEM and BSEI imaging, resulting in a better bond and much more slender interfacial zone than the ordinary concrete [14]. So, materials which are produced can be considered a two-phase composite material.

The purpose of the composites materials approach is to develop a model that will enable expression of average properties of the mixtures through properties and volume fractions of its constituents [1,11]. We look for the models to estimate the Young modulus for Lightweight Aggregate Concrete (LWAC) in terms of the properties and volume fractions of its constituents. These include the mortar matrix and the lightweight aggregate as reinforcing material. Before analyzing Lightweight Aggregate Concrete as a composite material, some assumptions must be considered.

First, the heterogeneous composite material (LWAC) is considered to be comprised of only two linear-elastic phases (the mortar and the lightweight aggregate). Second, the unit cell is assumed sufficiently large to account for the heterogeneity of the system, and the deferring geometry of the phases. However, it is extremely small so that the composite is described homogeneous on a macro scale $[10,15,16]$. Fig. 1 presents the models for an idealized unit cell of a two-phase composite material $[10,11,17]$.

The LWAC comprises a dispersed phase of lightweight aggregate with a Young's modulus $E_{\mathrm{g}}$ and volume fraction $\mathrm{V}_{\mathrm{g}}$ and a continuous phase of the mortar matrix, with a Young's modulus $\mathrm{E}_{\mathrm{m}}$ and volume fraction $\mathrm{V}_{\mathrm{m}}$. 
As explained by Gilormini and Brechert [18], the choice of a model is governed by several parameters including the geometry of the heterogonous medium, the mechanical contrast between the phases $\left(\mathrm{E}_{\mathrm{g}} / \mathrm{E}_{\mathrm{m}}\right)$ and the volume fraction of reinforcement $\left(\mathrm{V}_{\mathrm{g}}\right)$. Therefore, the equivalent homogenous behavior of LWAC depends of the characteristics of the mortar (matrix, phase $\mathrm{m}$ ) and lightweight aggregate (dispersed phase, phase $\mathrm{g}$ ).

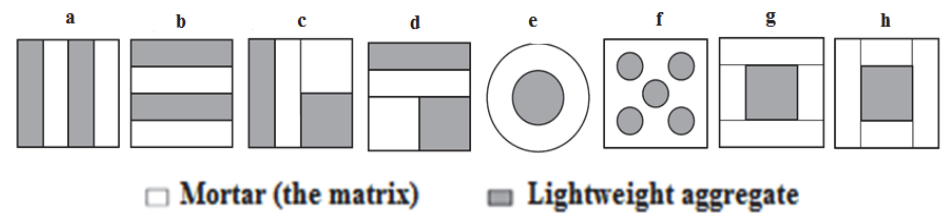

Figure 1: Composite models: (a) Voigt model, (b) Reuss model, (c) Popovics model, (d) Hirsch-Dougill model, (e) Hashin-Hansen model, (f) Maxwell model, (g) Counto1 model, (h) Counto2 model.

Voigt model [10, 19]:

Reuss model $[10,19]$ :

Popovics model [10, 20]:

Hirsch-Dougill model [10, 15, 21]:

$$
\mathrm{E}_{\mathrm{c}_{-} \text {Voigt }}=\mathrm{E}_{\mathrm{m}} \mathrm{V}_{\mathrm{m}}+\mathrm{E}_{\mathrm{g}} \mathrm{V}_{\mathrm{g}} \text {. }
$$

$$
\mathrm{E}_{\mathrm{c} \_ \text {Reuss }}=\frac{\mathrm{E}_{\mathrm{m}} \mathrm{E}_{\mathrm{g}}}{\mathrm{E}_{\mathrm{g}}+\mathrm{V}_{\mathrm{g}}\left(\mathrm{E}_{\mathrm{m}}-\mathrm{E}_{\mathrm{g}}\right)}
$$

Counto2 model [17]: $\mathrm{E}_{\mathrm{c}_{-} \text {Counto2 }}=\mathrm{E}_{\mathrm{m}}\left[1+\frac{\mathrm{V}_{\mathrm{g}}}{\frac{\mathrm{E}_{\mathrm{g}}}{\left(\mathrm{E}_{\mathrm{g}}-\mathrm{E}_{\mathrm{m}}\right)}-\sqrt{\mathrm{V}_{\mathrm{g}}}}\right]$.

Maxwell model (dispersed phase) $[10,15]:\left\{\begin{array}{c}\mathrm{E}_{\mathrm{c}_{-} \text {Maxwell }}=\mathrm{E}_{\mathrm{m}}\left[\frac{1+2 \mathrm{~V}_{\mathrm{g}}(\alpha-1) /(\alpha+2)}{1-\mathrm{V}_{\mathrm{g}}(\alpha-1) /(\alpha+2)}\right] \\ \alpha=\frac{\mathrm{E}_{\mathrm{g}}}{\mathrm{E}_{\mathrm{m}}}\end{array}\right.$.

Counto1 $\bmod [17,23]: \quad \mathrm{E}_{\mathrm{c}_{-} \text {Counto1 }}=\mathrm{E}_{\mathrm{m}}\left[1+\frac{\mathrm{V}_{\mathrm{g}}}{\sqrt{\mathrm{V}_{\mathrm{g}}}-\mathrm{V}_{\mathrm{g}}+\frac{\mathrm{E}_{\mathrm{m}}}{\left(\mathrm{E}_{\mathrm{g}}-\mathrm{E}_{\mathrm{m}}\right)}}\right]$.

Bache and Nepper-Christensen model [15, 24]: $\quad \mathrm{E}_{\mathrm{c}_{-} \text {Bache }}=\mathrm{E}_{\mathrm{m}} \mathrm{V}_{\mathrm{m}} \times \mathrm{E}_{\mathrm{g}} \mathrm{V}_{\mathrm{g}}$ 
The models of Voigt (Eq. and Reuss (Eq.2) provide the upper and lower bound of effective properties, respectively. It has been indicated [11, 19] that the upper bound relation of the parallel phase 'Voigt Model' might be applied as a first approximation to LWAC when $\mathrm{E}_{\mathrm{g}}<\mathrm{E}_{\mathrm{m}}$. However, the relation of the series phase 'Reuss model' validates the results of normal weight concrete with $\mathrm{E}_{\mathrm{g}}>\mathrm{E}_{\mathrm{m}}[11,19]$.

The biphasic models of Popovics (Eq. 3) and Hirsch-Dougill (Eq. 4) originally designed for composites with particles (like concrete) [25], propose elastic modulus of the composite by combining the Voigt and Reuss models. Hirsh [21] derived an equation to express the elastic modulus of concrete in terms of empirical constant, and also provided some experimental results for the elastic modulus of concrete with different aggregates.

The model composite spheres was introduced by Hashin [25]. This model consists of a gradation of size of spherical particles embedded in a continuous matrix [26]. Hansen [19] evolved mathematical models to predict the elastic modulus of composite materials based on the individual elastic modulus and volume portion of the components. From the concentric model, Hashin-Hansen model (Eq. 5) supposes that the Poisson ratios of all phases and the composite are equal $\left(v_{\mathrm{c}}=v_{\mathrm{m}}=0.2\right)[10,19]$.

The dispersed phase model "Maxwell model", Eq. (6), describes concrete as a dispersed phase composite material [10, 11]. As a concentric model [10], Zhou et al. [17] indicate that a more realistic Counto1 model (Eq.7) can be considered (Fig. 1g). Another version of Counto's model (Eq.8) [17] is presented in Fig. 1h. The strength-based Bache and NepperChristensen model (Eq. 9), gives a geometric average of component properties in relation to their volume fractions $V_{m}$ and $\mathrm{V}_{\mathrm{g}}$. This is a mathematical model with no physical meaning [17].

\section{Experimental data from published literature}

In this section, the bibliography data for different Lightweight Aggregate Concrete (LWAC) tested experimentally by De Larrard [7], Yang and Huang [8] and Ke Y et al. [9] are compiled in Tabs. 1, 2 and 3, respectively. The mechanical properties $\mathrm{E}_{\mathrm{m}}, \mathrm{E}_{\mathrm{g}}$ are the Young's modulus of the matrix (mortar: phase $\mathrm{m}$ ) and lightweight aggregate (dispersed phase, phase g), respectively. The Young's modulus of the composite obtained experimentally by De Larrard [7], Yang and Huang [8] and Ke $\mathrm{Y}$ et al. [9] are $E_{\text {exp_De Larrard }}, E_{\text {exp_Yang }}$ and $\mathrm{E}_{\text {exp_Ke }}$ respectively.

For LWAC test results by De Larrard [7] compiled in Tab. 1, it can be seen that the volume fraction Vg (the volume fraction of the lightweight aggregate) varies from $25.5 \%$ to $47.8 \%$ and that the contrast of the characteristics of the phases $\mathrm{E}_{\mathrm{g}} / \mathrm{E}_{\mathrm{m}}$ varies between $27.74 \%$ and $95 \%$ except for four types of concretes for which this ratio exceeds 1 because of a very low value of $\mathrm{E}_{\mathrm{m}}\left(\mathrm{E}_{\mathrm{g}}>\mathrm{E}_{\mathrm{m}}\right)$.

In their experimental program Yang and Huang [8] have tested three types of artificial coarse aggregates with Young's modulus of $6.01,7.97$ and $10.48 \mathrm{GPa}$ made of cement and fly ash with various combinations through a cold-pelletizing process. Each type of aggregate was mixed with four types of mortar matrices with a Young's modulus of 29.330, 28.130, 26.440 and $24.870 \mathrm{GPa}$. This corresponds to a contrast ratio $\mathrm{E}_{\mathrm{g}} / \mathrm{E}_{\mathrm{m}}$ between the two phases ranging from $20.49 \%$ to $42.14 \%$. By supposing a Poisson's ratio of 0.2 , the strength of coarse aggregate was computed from the elastic moduli of the components and the strength of concrete. The rate of lightweight aggregate volume fraction $\mathrm{Vg}$ was between 18\% and $36 \%$, the diameter of the gold aggregates assumed as spherical for all concretes tested had a d/D ratio in the order of $(5 / 10) \mathrm{mm}$ (Tab. 2). In their study, concrete was considered as a composite material in which coarse aggregate were embedded in a matrix of hardened mortar.

In the experimental study of Ke $\mathrm{Y}$ et al. [9], five LWAs are used: three expanded clay aggregates (A) of quasi-spherical shape (0/4 650A, 4/10 550A, 4/10 430 A) and two aggregates of expanded shale (S) of irregular shape (4/10 520S, 4/8 750 S). The three used matrices (called M8, M9 and M10) are made of Portland cement mortar CEM I 52.5 and normal sand $0 / 2 \mathrm{~mm}$. Normal, high performance (HP) and very high performance (VHP) mortar matrices, were utilized for the realization of the concrete specimens tested by Ke Y et al. [9]. In their work, the volume fraction of aggregate was $0 \%$ (mortar), $12.5 \%, 25 \%, 37.5 \%$ and $45 \%$ with a contrast of the properties varying from $12.26 \%$ to $69.61 \%$. The Young's modulus of the three mortar matrices were experimentally determined as 28.6, 33.2 and 35.4 GPa for M8, M9 and M10, respectively, as seen in Tab. 3 [9]. They correspond to a normal, HP and VHP matrix, respectively [9]. Mechanical properties of the lightweight aggregate are shown in Tab. 4 [9].

The elastic modulus of LWAC is estimated by utilizing some composite material models $E_{c_{-} \text {anal }}$ like Popovics, HirschDougill, Hashin-Hansen, Maxwell, Counto1, Counto2, and Bache and Nepper-Christensen (Eqs.(2)-(9)). 
This Study try to figure out that these composite material models, mentioned above, got reliable prediction abilities for the modulus of elasticity of LWAC. The modulus of elasticity values were predicted utilizing the composite models and then, the predicted results were compared to the experimental results of De Larrard [7], Yang and Huang [8] and Ke Y et al. [9] respectively.

\begin{tabular}{|c|c|c|c|c|c|}
\hline $\begin{array}{l}\text { Ref. } \\
\text { grav. }\end{array}$ & $\begin{array}{l}\mathrm{d} / \mathrm{D} \\
(\mathrm{mm})\end{array}$ & $\mathrm{V}_{\mathrm{g}}$ & $\begin{array}{c}\mathrm{E}_{\mathrm{g}} \\
(\mathrm{GPa})\end{array}$ & $\begin{array}{c}\mathrm{E}_{\mathrm{m}} \\
(\mathrm{GPa})\end{array}$ & $\begin{array}{c}E_{\text {exp_De Larrard }} \\
\quad(\mathrm{GPa})\end{array}$ \\
\hline 8 Argi 16 & 4-12 & 0.414 & 8 & 25.2 & 15.6 \\
\hline 8 Isol S & $3.15-8$ & 0.414 & 13.1 & 25.2 & 19.2 \\
\hline 8 Leca 7j. & $4-10$ & 0.414 & 7.6 & 23.5 & 14.1 \\
\hline 8 Leca $28 j$. & $4-10$ & 0.414 & 7.6 & 25.2 & 15.7 \\
\hline 8 Surex 675 & $6.3-10$ & 0.414 & 16.2 & 25.2 & 21 \\
\hline 8 Galex 7j. & 3-8 & 0.425 & 33 & 20.6 & 25.5 \\
\hline 8 Galex 28j. & $3-8$ & 0.425 & 33 & 21.9 & 25.8 \\
\hline 9 Schiste $15 j$. & - & 0.391 & 21 & 24.9 & 23.9 \\
\hline 9 Schiste $28 \mathrm{j}$. & - & 0.391 & 21 & 25.6 & 23.4 \\
\hline 9 Leca $1 j$. & $4-10$ & 0.414 & 8.6 & 11 & 10 \\
\hline 9 Leca $2 j$. & $4-10$ & 0.414 & 8.6 & 15 & 12 \\
\hline 9 Leca $7 j$. & $4-10$ & 0.414 & 8.6 & 20 & 13.9 \\
\hline 9 Leca $28 j$. & $4-10$ & 0.414 & 8.6 & 25.2 & 16 \\
\hline 9 Leca 90j. & $4-10$ & 0.414 & 8.6 & 31 & 17.8 \\
\hline 9 Surex $1 j$. & $6.3-10$ & 0.414 & 19 & 11 & 14.6 \\
\hline 9 Surex 2j. & $6.3-10$ & 0.414 & 19 & 15 & 17.1 \\
\hline 9 Surex $7 j$. & $6.3-10$ & 0.414 & 19 & 20 & 20.6 \\
\hline 9 Surex $28 j$. & $6.3-10$ & 0.414 & 19 & 25.2 & 22.3 \\
\hline 9 Surex $90 j$. & $6.3-10$ & 0.414 & 19 & 31 & 22.6 \\
\hline 3 LWC1 Crush & $10-20$ & 0.403 & 13.6 & 33.2 & 23 \\
\hline 3 LWC1 Crush & $10-20$ & 0.403 & 13.6 & 33.2 & 24.3 \\
\hline 3 LWC1 Pellet & $5-20$ & 0.414 & 14 & 32.8 & 22.7 \\
\hline 3 LWC1 Pellet & $5-20$ & 0.414 & 14 & 32.8 & 24.3 \\
\hline 3 HSLWC Pel. & $5-20$ & 0.255 & 14 & 38.5 & 27.6 \\
\hline 3 HSLWC Pel. & $5-20$ & 0.255 & 14 & 38.5 & 28.3 \\
\hline 1 Liapor & $0-16$ & 0.473 & 21.5 & 29.7 & 25.5 \\
\hline 2 Liapor & $4-16$ & 0.432 & 21.5 & 27.9 & 24.8 \\
\hline 16 Javron & $4-10$ & 0.463 & 16 & 23.8 & 19.7 \\
\hline $16 \mathrm{G} / \mathrm{S}-0.2$ & $4-10$ & 0.443 & 16 & 24.6 & 19.9 \\
\hline $16 \mathrm{G} / \mathrm{S}+0.2$ & $4-10$ & 0.478 & 16 & 23.1 & 19.7 \\
\hline $16 \mathrm{EAU}+$ & $4-10$ & 0.473 & 16 & 26.6 & 20.9 \\
\hline $16 \mathrm{EAU}-$ & $4-10$ & 0.453 & 16 & 21.1 & 18.1 \\
\hline
\end{tabular}

Table 1: Characteristics of LWAC tested by De Larrard [7].

\begin{tabular}{cccccc}
\hline $\begin{array}{c}\text { Ref. } \\
\text { grav. }\end{array}$ & $\begin{array}{c}\mathrm{d} / \mathrm{D} \\
(\mathrm{mm})\end{array}$ & $\mathrm{V}_{\mathrm{g}}$ & $\begin{array}{c}\mathrm{E}_{\mathrm{g}} \\
(\mathrm{GPa})\end{array}$ & $\begin{array}{c}\mathrm{E}_{\mathrm{m}} \\
(\mathrm{GPa})\end{array}$ & $\begin{array}{c}\mathrm{E}_{\text {exp_Yang }} \\
(\mathrm{GPa})\end{array}$ \\
$\mathrm{A} 3$ & & 0.18 & 6.01 & 29.33 & 23.020 \\
$\mathrm{~A} 4$ & & 0.24 & 6.01 & 28.13 & 20.600 \\
A5 & & 0.30 & 6.01 & 26.44 & 18.210 \\
A6 & & 0.36 & 6.01 & 24.87 & 15.800 \\
B3 & & 0.18 & 7.97 & 29.33 & 23.790 \\
B4 & $5-10$ & 0.24 & 7.97 & 28.13 & 21.530 \\
B5 & & 0.30 & 7.97 & 26.44 & 19.010 \\
B6 & & 0.36 & 7.97 & 24.87 & 17.220 \\
C3 & & 0.18 & 10.48 & 29.33 & 24.660 \\
C4 & & 0.24 & 10.48 & 28.13 & 22.580 \\
C5 & & 0.30 & 10.48 & 26.44 & 20.320 \\
C6 & & 0.36 & 10.48 & 24.87 & 18.650 \\
\hline
\end{tabular}

Table 2: Characteristics of LWAC tested by Chung-Chia Yang and Ran Huang [8]. 


\begin{tabular}{cllllll}
\hline & \multicolumn{1}{c}{$\mathrm{E}_{\text {exp_Ke }}$} & $\mathrm{E}_{0} \mathrm{Ke}$ & $\mathrm{E}_{0.125} \mathrm{Ke}$ & $\mathrm{E}_{0.250}{ }^{\mathrm{Ke}}$ & $\mathrm{E}_{0.375} \mathrm{Ke}$ & $\mathrm{E}_{0.450} \mathrm{Ke}^{\mathrm{Ke}}$ \\
& 0/4 650A & 28.588 & 23.539 & 20.665 & 16.743 & 15.669 \\
\multirow{4}{*}{$\mathrm{M} 8$} & 4/10 550A & & 26.157 & 21.680 & 17.900 & 16.606 \\
& 4/10 430A & & 24.900 & 21.391 & 17.293 & 15.699 \\
& 4/10 520S & & 25.135 & 22.471 & 19.428 & 18.286 \\
& 4/8 750S & & 27.367 & 26.262 & 25.281 & 24.324 \\
& 0/4 650A & 33.183 & 29.396 & 23.712 & 19.871 & 17.175 \\
M9 & 4/10 550A & & 29.159 & 24.934 & 21.358 & 19.696 \\
& 4/10 430A & & 27.568 & 23.778 & 20.818 & 18.935 \\
& 4/10 520S & & 29.480 & 26.521 & 22.188 & 20.184 \\
& 4/8 750S & & 31.931 & 30.987 & 30.146 & 29.311 \\
& 0/4 650A & 35.397 & 31.147 & 26.753 & 22.427 & 20.346 \\
& 4/10 550A & & 32.089 & 27.991 & 23.684 & 21.724 \\
& 4/10 430A & & 30.220 & 26.033 & 22.296 & 20.082 \\
& 4/10 520S & & 32.783 & 27.998 & 24.340 & 22.024 \\
& 4/8 750S & & 34.213 & 33.845 & 32.945 & 33.002 \\
\hline
\end{tabular}

Table 3: Characteristics of LWAC tested by Ke Y et al. [9] (GPa).

\begin{tabular}{cccccc}
\hline \multirow{2}{*}{ LWA } & $0 / 4$ & $4 / 10$ & $4 / 10$ & $4 / 10$ & $4 / 8$ \\
\multirow{2}{*}{$\mathrm{Eg}$} & $650 \mathrm{~A}$ & $550 \mathrm{~A}$ & $430 \mathrm{~A}$ & $520 \mathrm{~S}$ & $750 \mathrm{~S}$ \\
\hline
\end{tabular}

Table 4: Mechanical properties of lightweight aggregate tested by Ke Y et al. [9] (GPa).

\section{RESULTS AND DISCUSSIONS}

\section{Comparative analysis}

omparison between the estimative results of effective elastic modulus of LWAC obtained as a result of calculations of the Eqns. (2-9) and those of experimental data have been presented in Tabs. 5, 6 and 7 respectively.

A confrontation of LWAC Young's modulus between experimental results in $[7,8,9]$ and the predictions of 07 composite models material models are shown in Fig. 2, Fig. 3 and Fig. 4 respectively.

The differences between the various predictive composite models and the experimental results in $[7,8,9]$ have been computed according to the proportion of reinforcement Vg in LWAC. When the volume fraction of aggregates Vg grows, the errors between the predictions and the experimental results increase for all composite material models. Since the weakest component of LWAC is not the cement matrix but the lightweight aggregates, the effect of volume fraction of lightweight aggregate on Young's modulus of LWAC is very clear. The increase in the volume fraction of lightweight aggregates Vg substantially reduces the Young's modulus of the LWAC.

To compare the experimental and predicted Young's modulus of LWAC, the error percentage $|\Delta \mathrm{E}|$. is determined using the following expression:

$$
\Delta \mathrm{E}(\%)=\left(\frac{\mathrm{E}_{\mathrm{c} \_ \text {anal }_{1}}-\mathrm{E}_{\text {exp }}}{\mathrm{E}_{\text {exp }}}\right) \times 100
$$

$|\Delta \mathrm{E}|=\operatorname{Abs}(\Delta \mathrm{E})$, Absolute value of $\Delta \mathrm{E}$

It appears for first time that all models are generally suitable for predicting the modulus of elasticity of the LWAC.

Tabs. 8-9-10 give the error percentages of the composite material models and experimental results in $[7,8,9]$ respectively. In order to choose the models which have good performances, the error percentages below $10 \%$ are chosen as desired range and the model's error percentages below this value are indicated in bold. Therefore, the models which verified this condition have been underlined. 


\begin{tabular}{|c|c|c|c|c|c|c|c|c|}
\hline $\begin{array}{l}\text { Ref. } \\
\text { grav. }\end{array}$ & E $_{\text {exp_De Larrard }}$ & $\mathrm{E}_{\mathrm{c} \_ \text {Popovics }}$ & $\mathrm{E}_{\mathrm{c} \_ \text {Hirsch }}$ & $\mathrm{E}_{\mathrm{c} \_ \text {Hashin }}$ & $\mathrm{E}_{\mathrm{c} \_ \text {Maxwell }}$ & $\mathrm{E}_{\mathrm{c} \_ \text {Counto1 }}$ & $\mathrm{E}_{\mathrm{c} \_ \text {Counto2 }}$ & $\mathrm{E}_{\mathrm{c} \_ \text {Bache }}$ \\
\hline 8 Argi 16 & 15.6 & 15.71 & 15.35 & 16.30 & 16.98 & 16.76 & 15.79 & 15.67 \\
\hline 8 Isol S & 19.2 & 19.21 & 19.16 & 19.37 & 19.67 & 19.57 & 19.16 & 19.22 \\
\hline 8 Leca $7 j$. & 14.1 & 14.76 & 14.44 & 15.29 & 15.91 & 15.71 & 14.82 & 14.73 \\
\hline 8 Leca $28 j$. & 15.7 & 15.39 & 14.98 & 16.04 & 16.76 & 16.52 & 15.50 & 15.34 \\
\hline 8 Surex 675 & 21 & 20.98 & 20.97 & 21.04 & 21.19 & 21.14 & 20.93 & 20.99 \\
\hline 8 Galex 7j. & 25.5 & 25.19 & 25.17 & 25.09 & 25.32 & 25.24 & 24.96 & 25.17 \\
\hline 8 Galex 28j. & 25.8 & 26.09 & 26.07 & 26.02 & 26.20 & 26.13 & 25.91 & 26.07 \\
\hline 9 Schiste $15 \mathrm{j}$. & 23.9 & 23.29 & 23.29 & 23.30 & 23.33 & 23.32 & 23.28 & 23.30 \\
\hline 9 Schiste $28 \mathrm{j}$. & 23.4 & 23.69 & 23.69 & 23.70 & 23.73 & 23.72 & 23.67 & 23.69 \\
\hline 9 Leca $1 j$. & 10 & 9.93 & 9.93 & 9.94 & 9.96 & 9.95 & 9.92 & 9.93 \\
\hline 9 Leca $2 j$. & 12 & 11.91 & 11.89 & 11.97 & 12.11 & 12.06 & 11.87 & 11.91 \\
\hline 9 Leca $7 j$. & 13.9 & 14.10 & 14.00 & 14.33 & 14.69 & 14.57 & 14.08 & 14.10 \\
\hline 9 Leca 28j. & 16 & 16.17 & 15.88 & 16.68 & 17.31 & 17.10 & 16.22 & 16.15 \\
\hline 9 Leca 90j. & 17.8 & 18.32 & 17.69 & 19.24 & 20.20 & 19.88 & 18.51 & 18.23 \\
\hline 9 Surex $1 j$. & 14.6 & 13.82 & 13.80 & 13.73 & 13.90 & 13.84 & 13.63 & 13.79 \\
\hline 9 Surex $2 j$. & 17.1 & 16.54 & 16.54 & 16.54 & 16.57 & 16.56 & 16.51 & 16.54 \\
\hline 9 Surex 7j. & 20.6 & 19.58 & 19.58 & 19.58 & 19.58 & 19.58 & 19.58 & 19.58 \\
\hline 9 Surex 28j. & 22.3 & 22.42 & 22.41 & 22.43 & 22.50 & 22.48 & 22.39 & 22.42 \\
\hline 9 Surex 90j. & 22.6 & 25.30 & 25.28 & 25.40 & 25.63 & 25.55 & 25.24 & 25.31 \\
\hline 3 LWC1 Crush & 23 & 23.15 & 22.95 & 23.61 & 24.25 & 24.05 & 23.13 & 23.17 \\
\hline 3 LWC1 Crush & 24.3 & 23.15 & 22.95 & 23.61 & 24.25 & 24.05 & 23.13 & 23.17 \\
\hline 3 LWC1 Pellet & 22.7 & 23.05 & 22.88 & 23.45 & 24.04 & 23.84 & 23.02 & 23.06 \\
\hline 3 LWC1 Pellet & 24.3 & 23.05 & 22.88 & 23.45 & 24.04 & 23.84 & 23.02 & 23.06 \\
\hline 3 HSLWC Pel. & 27.6 & 29.44 & 29.17 & 30.31 & 31.08 & 31.07 & 29.38 & 29.75 \\
\hline 3 HSLWC Pel. & 28.3 & 29.44 & 29.17 & 30.31 & 31.08 & 31.07 & 29.38 & 29.75 \\
\hline 1 Liapor & 25.5 & 25.49 & 25.49 & 25.52 & 25.62 & 25.58 & 25.46 & 25.49 \\
\hline 2 Liapor & 24.8 & 24.93 & 24.93 & 24.94 & 25.01 & 24.98 & 24.90 & 24.93 \\
\hline 16 Javron & 19.7 & 19.80 & 19.80 & 19.84 & 19.96 & 19.91 & 19.77 & 19.80 \\
\hline $16 \mathrm{G} / \mathrm{S}-0.2$ & 19.9 & 20.33 & 20.32 & 20.38 & 20.53 & 20.47 & 20.29 & 20.33 \\
\hline $16 \mathrm{G} / \mathrm{S}+0.2$ & 19.7 & 19.38 & 19.38 & 19.41 & 19.51 & 19.47 & 19.35 & 19.38 \\
\hline $16 \mathrm{EAU}+$ & 20.9 & 20.92 & 20.90 & 21.00 & 21.21 & 21.12 & 20.87 & 20.92 \\
\hline $16 \mathrm{EAU}-$ & 18.1 & 18.61 & 18.61 & 18.63 & 18.68 & 18.66 & 18.59 & 18.61 \\
\hline
\end{tabular}

Table 5: Modulus of elasticity of LWAC predicted by various composite models compared with the experimental results of De Larrard $[7](\mathrm{GPa})$.

\begin{tabular}{|c|c|c|c|c|c|c|c|c|}
\hline $\begin{array}{l}\text { Ref. } \\
\text { grav. }\end{array}$ & $\mathrm{E}_{\text {exp_Yang }}$ & $\mathrm{E}_{\mathrm{c} \_ \text {Popovics }}$ & $\mathrm{E}_{\mathrm{c} \_ \text {Hirsch }}$ & $\mathrm{E}_{\mathrm{c} \_ \text {Hashin }}$ & $\mathrm{E}_{\mathrm{c} \_ \text {Maxwell }}$ & $\mathrm{E}_{\mathrm{c} \_ \text {Counto1 }}$ & $\mathrm{E}_{\mathrm{c} \_ \text {Counto2 }}$ & $\mathrm{E}_{\mathrm{c} \_ \text {Bache }_{1}}$ \\
\hline A3 & 23.020 & 21.201 & 20.471 & 23.102 & 23.967 & 24.121 & 21.589 & 22.049 \\
\hline A4 & 20.600 & 18.879 & 18.055 & 20.559 & 21.501 & 21.523 & 19.265 & 19.422 \\
\hline A5 & 18.210 & 16.701 & 15.920 & 18.039 & 18.963 & 18.860 & 17.018 & 16.953 \\
\hline A6 & 15.800 & 14.879 & 14.190 & 15.905 & 16.770 & 16.570 & 15.124 & 14.915 \\
\hline B3 & 23.790 & 22.635 & 22.276 & 23.848 & 24.530 & 24.653 & 22.709 & 23.198 \\
\hline B4 & 21.530 & 20.398 & 19.987 & 21.481 & 22.218 & 22.236 & 20.504 & 20.784 \\
\hline B5 & 19.010 & 18.248 & 17.863 & 19.106 & 19.820 & 19.739 & 18.340 & 18.451 \\
\hline B6 & 17.220 & 16.445 & 16.112 & 17.095 & 17.754 & 17.600 & 16.515 & 16.510 \\
\hline C3 & 24.660 & 24.047 & 23.898 & 24.723 & 25.214 & 25.305 & 23.944 & 24.370 \\
\hline $\mathrm{C} 4$ & 22.580 & 21.963 & 21.794 & 22.568 & 23.093 & 23.106 & 21.900 & 22.195 \\
\hline $\mathrm{C} 5$ & 20.320 & 19.900 & 19.746 & 20.369 & 20.867 & 20.810 & 19.854 & 20.030 \\
\hline C6 & 18.650 & 18.166 & 18.039 & 18.512 & 18.960 & 18.854 & 18.130 & 18.221 \\
\hline
\end{tabular}

Table 6: Modulus of elasticity of LWAC predicted by various composite models compared with the experimental results of Yang and Huang $[8](\mathrm{GPa})$. 
M.F. Bouali et alii, Frattura ed Integrità Strutturale, 52 (2020) 82-97; DOI: 10.3221/IGF-ESIS.52.07

\begin{tabular}{|c|c|c|c|c|c|c|c|c|}
\hline $\begin{array}{l}\text { Ref. } \\
\text { grav. }\end{array}$ & $\mathrm{E}_{\text {exp_Ke }}$ & E $_{\text {c_Popovics }}$ & $\mathrm{E}_{\mathrm{c} \_ \text {Hirsch }}$ & $\mathrm{E}_{\mathrm{c} \_ \text {Hashin }}$ & $\mathrm{E}_{\mathrm{c} \_ \text {Maxwell }}$ & $\mathrm{E}_{\mathrm{c} \_ \text {Counto } 1}$ & $\mathrm{E}_{\mathrm{c} \_ \text {Counto2 }}$ & $\mathrm{E}_{\mathrm{c} \_ \text {Bache }}$ \\
\hline \multirow{5}{*}{$\begin{array}{l}\text { M8 } \\
0 / 4650 \text { A }\end{array}$} & 28.588 & 28.588 & 28.588 & 28.588 & 28.588 & 28.588 & 28.588 & 28.588 \\
\hline & 23.539 & 23.182 & 22.870 & 24.522 & 25.101 & 25.303 & 23.253 & 23.921 \\
\hline & 20.665 & 19.563 & 18.903 & 20.996 & 21.886 & 21.886 & 19.833 & 20.016 \\
\hline & 16.743 & 16.762 & 15.954 & 17.908 & 18.912 & 18.652 & 17.044 & 16.748 \\
\hline & 15.669 & 15.308 & 14.504 & 16.234 & 17.233 & 16.845 & 15.556 & 15.050 \\
\hline \multirow{5}{*}{$\begin{array}{l}\text { M8 } \\
4 / 10550 \mathrm{~A}\end{array}$} & 28.588 & 28.588 & 28.588 & 28.588 & 28.588 & 28.588 & 28.588 & 28.588 \\
\hline & 26.157 & 23.132 & 22.810 & 24.499 & 25.084 & 25.288 & 23.215 & 23.886 \\
\hline & 21.680 & 19.499 & 18.820 & 20.956 & 21.855 & 21.855 & 19.781 & 19.957 \\
\hline & 17.900 & 16.693 & 15.863 & 17.857 & 18.870 & 18.607 & 16.984 & 16.675 \\
\hline & 16.606 & 15.237 & 14.413 & 16.176 & 17.185 & 16.793 & 15.492 & 14.971 \\
\hline \multirow{5}{*}{$\begin{array}{l}\text { M8 } \\
4 / 10430 \mathrm{~A}\end{array}$} & 28.588 & 28.588 & 28.588 & 28.588 & 28.588 & 28.588 & 28.588 & 28.588 \\
\hline & 24.900 & 21.195 & 20.297 & 23.769 & 24.561 & 24.828 & 21.878 & 22.586 \\
\hline & 21.391 & 17.227 & 15.597 & 19.699 & 20.895 & 20.895 & 18.062 & 17.845 \\
\hline & 17.293 & 14.366 & 12.534 & 16.216 & 17.543 & 17.203 & 15.041 & 14.099 \\
\hline & 15.699 & 12.906 & 11.142 & 14.357 & 15.667 & 15.162 & 13.450 & 12.240 \\
\hline \multirow{5}{*}{$\begin{array}{l}\text { M8 } \\
4 / 10520 \mathrm{~S}\end{array}$} & 28.588 & 28.588 & 28.588 & 28.588 & 28.588 & 28.588 & 28.588 & 28.588 \\
\hline & 25.135 & 22.939 & 22.576 & 24.414 & 25.022 & 25.233 & 23.067 & 23.752 \\
\hline & 22.471 & 19.253 & 18.499 & 20.808 & 21.740 & 21.740 & 19.583 & 19.733 \\
\hline & 19.428 & 16.429 & 15.516 & 17.662 & 18.711 & 18.439 & 16.756 & 16.395 \\
\hline & 18.286 & 14.967 & 14.063 & 15.959 & 17.002 & 16.597 & 15.250 & 14.669 \\
\hline \multirow{5}{*}{$\begin{array}{l}\text { M8 } \\
4 / 10750 \mathrm{~S}\end{array}$} & 28.588 & 28.588 & 28.588 & 28.588 & 28.588 & 28.588 & 28.588 & 28.588 \\
\hline & 27.367 & 27.305 & 27.304 & 27.335 & 27.396 & 27.421 & 27.236 & 27.322 \\
\hline & 26.262 & 26.095 & 26.091 & 26.137 & 26.237 & 26.237 & 26.027 & 26.113 \\
\hline & 25.281 & 24.948 & 24.942 & 24.988 & 25.110 & 25.077 & 24.895 & 24.957 \\
\hline & 24.324 & 24.286 & 24.280 & 24.322 & 24.448 & 24.397 & 24.244 & 24.288 \\
\hline \multirow{5}{*}{$\begin{array}{l}\text { M9 } \\
0 / 4650 \mathrm{~A}\end{array}$} & 33.183 & 33.183 & 33.183 & 33.183 & 33.183 & 33.183 & 33.183 & 33.183 \\
\hline & 29.396 & 26.167 & 25.636 & 28.147 & 28.904 & 29.166 & 26.435 & 27.253 \\
\hline & 23.712 & 21.778 & 20.708 & 23.821 & 24.978 & 24.978 & 22.283 & 22.383 \\
\hline & 19.871 & 18.468 & 17.195 & 20.065 & 21.363 & 21.028 & 18.937 & 18.384 \\
\hline & 17.175 & 16.763 & 15.512 & 18.040 & 19.328 & 18.829 & 17.160 & 16.336 \\
\hline \multirow{5}{*}{$\begin{array}{l}\text { M9 } \\
4 / 10550 \mathrm{~A}\end{array}$} & 33.183 & 33.183 & 33.183 & 33.183 & 33.183 & 33.183 & 33.183 & 33.183 \\
\hline & 29.159 & 26.108 & 25.562 & 28.123 & 28.887 & 29.151 & 26.392 & 27.214 \\
\hline & 24.934 & 21.707 & 20.611 & 23.780 & 24.947 & 24.947 & 22.228 & 22.318 \\
\hline & 21.358 & 18.394 & 17.093 & 20.012 & 21.320 & 20.982 & 18.874 & 18.303 \\
\hline & 19.696 & 16.688 & 15.410 & 17.981 & 19.279 & 18.776 & 17.094 & 16.250 \\
\hline \multirow{5}{*}{$\begin{array}{l}\text { M9 } \\
4 / 10430 \mathrm{~A}\end{array}$} & 33.183 & 33.183 & 33.183 & 33.183 & 33.183 & 33.183 & 33.183 & 33.183 \\
\hline & 27.568 & 23.852 & 22.477 & 27.365 & 28.353 & 28.684 & 24.953 & 25.733 \\
\hline & 23.778 & 19.220 & 16.848 & 22.485 & 23.970 & 23.970 & 20.430 & 19.955 \\
\hline & 20.818 & 15.934 & 13.338 & 18.333 & 19.975 & 19.555 & 16.871 & 15.475 \\
\hline & 18.935 & 14.259 & 11.782 & 16.127 & 17.743 & 17.121 & 15.001 & 13.285 \\
\hline \multirow{5}{*}{$\begin{array}{l}\text { M9 } \\
4 / 10520 \mathrm{~S}\end{array}$} & 33.183 & 33.183 & 33.183 & 33.183 & 33.183 & 33.183 & 33.183 & 33.183 \\
\hline & 29.480 & 25.881 & 25.274 & 28.034 & 28.824 & 29.095 & 26.232 & 27.060 \\
\hline & 26.521 & 21.435 & 20.234 & 23.627 & 24.830 & 24.830 & 22.020 & 22.067 \\
\hline & 22.188 & 18.113 & 16.699 & 19.812 & 21.158 & 20.811 & 18.638 & 17.996 \\
\hline & 20.184 & 16.405 & 15.021 & 17.759 & 19.093 & 18.577 & 16.845 & 15.923 \\
\hline \multirow{5}{*}{$\begin{array}{l}\text { M9 } \\
4 / 10750 \mathrm{~S}\end{array}$} & 33.183 & 33.183 & 33.183 & 33.183 & 33.183 & 33.183 & 33.183 & 33.183 \\
\hline & 31.931 & 31.075 & 31.069 & 31.170 & 31.303 & 31.355 & 30.943 & 31.128 \\
\hline & 30.987 & 29.150 & 29.133 & 29.276 & 29.493 & 29.493 & 29.031 & 29.201 \\
\hline & 30.146 & 27.371 & 27.346 & 27.490 & 27.749 & 27.679 & 27.287 & 27.393 \\
\hline & 29.311 & 26.362 & 26.335 & 26.466 & 26.732 & 26.626 & 26.298 & 26.363 \\
\hline \multirow{5}{*}{$\begin{array}{l}\text { M10 } \\
0 / 4650 \text { A }\end{array}$} & 35.397 & 35.397 & 35.397 & 35.397 & 35.397 & 35.397 & 35.397 & 35.397 \\
\hline & 31.147 & 27.567 & 26.907 & 29.889 & 30.735 & 31.026 & 27.953 & 28.838 \\
\hline & 26.753 & 22.816 & 21.515 & 25.176 & 26.466 & 26.466 & 23.452 & 23.494 \\
\hline & 22.427 & 19.271 & 17.742 & 21.098 & 22.541 & 22.169 & 19.839 & 19.141 \\
\hline & 20.346 & 17.450 & 15.953 & 18.905 & 20.334 & 19.781 & 17.925 & 16.926 \\
\hline M10 & 35.397 & 35.397 & 35.397 & 35.397 & 35.397 & 35.397 & 35.397 & 35.397 \\
\hline $4 / 10550 \mathrm{~A}$ & 32.089 & 27.504 & 26.826 & 29.865 & 30.718 & 31.011 & 27.909 & 28.796 \\
\hline
\end{tabular}


M.F. Bouali et alii, Frattura ed Integrità Strutturale, 52 (2020) 82-97; DOI: 10.3221/IGF-ESIS.52.07

\begin{tabular}{lllllllll}
\hline & 27.991 & 22.742 & 21.411 & 25.135 & 26.434 & 26.434 & 23.396 & 23.426 \\
& 23.684 & 19.195 & 17.633 & 21.045 & 22.497 & 22.123 & 19.776 & 19.057 \\
& 21.724 & 17.373 & 15.847 & 18.845 & 20.285 & 19.727 & 17.858 & 16.837 \\
\hline & 35.397 & 35.397 & 35.397 & 35.397 & 35.397 & 35.397 & 35.397 & 35.397 \\
M10 & 30.220 & 25.099 & 23.460 & 29.096 & 30.180 & 30.541 & 26.427 & 27.229 \\
$4 / 10430 \mathrm{~A}$ & 26.033 & 20.162 & 17.394 & 23.825 & 25.451 & 25.451 & 21.564 & 20.946 \\
& 22.296 & 16.680 & 13.683 & 19.351 & 21.146 & 20.687 & 17.748 & 16.112 \\
& 20.082 & 14.904 & 12.055 & 16.977 & 18.742 & 18.063 & 15.746 & 13.766 \\
\hline & 35.397 & 35.397 & 35.397 & 35.397 & 35.397 & 35.397 & 35.397 & 35.397 \\
M10 & 32.783 & 27.261 & 26.510 & 29.775 & 30.654 & 30.954 & 27.743 & 28.634 \\
& 27.998 & 22.459 & 21.007 & 24.980 & 26.316 & 26.316 & 23.183 & 23.163 \\
& 24.340 & 18.906 & 17.218 & 20.843 & 22.334 & 21.950 & 19.536 & 18.737 \\
& 22.024 & 17.085 & 15.439 & 18.621 & 20.098 & 19.527 & 17.606 & 16.498 \\
\hline & 35.397 & 35.397 & 35.397 & 35.397 & 35.397 & 35.397 & 35.397 & 35.397 \\
410 S & 34.123 & 32.858 & 32.847 & 33.001 & 33.176 & 33.244 & 32.695 & 32.938 \\
& 33.845 & 30.576 & 30.546 & 30.762 & 31.047 & 31.047 & 30.437 & 30.651 \\
& 32.945 & 28.491 & 28.449 & 28.665 & 29.002 & 28.912 & 28.398 & 28.522 \\
& 33.002 & 27.317 & 27.273 & 27.469 & 27.815 & 27.677 & 27.249 & 27.316 \\
\hline
\end{tabular}

Table 7: Modulus of elasticity of LWAC predicted by various composite models compared with the experimental results of Ke Y et al. [9](GPa).

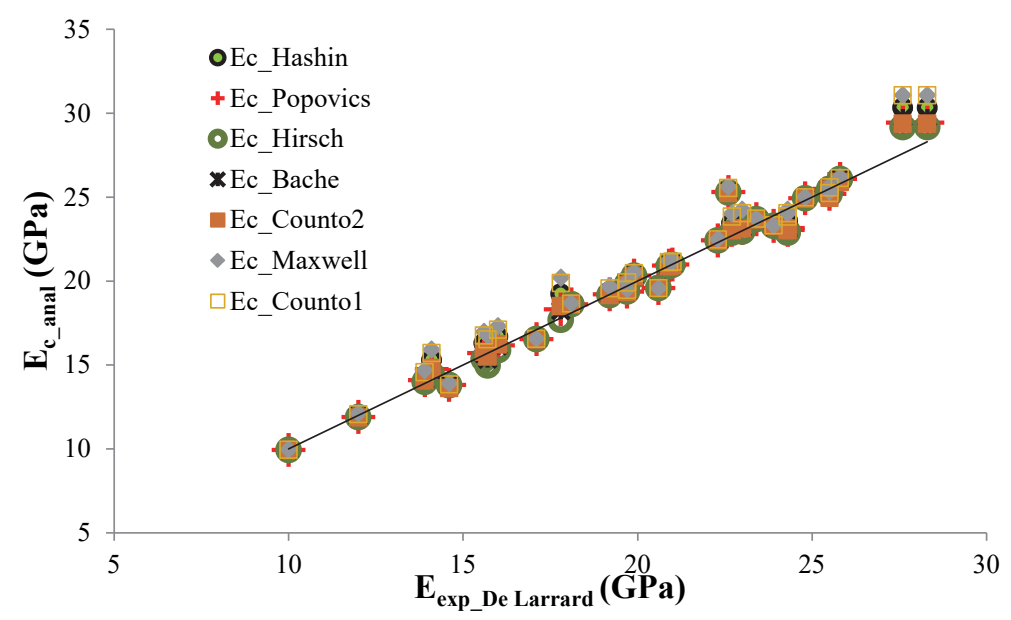

Figure 2: Confrontation of LWAC Young's modulus between experimental results in [7] and the predictions of 07 composite material models.

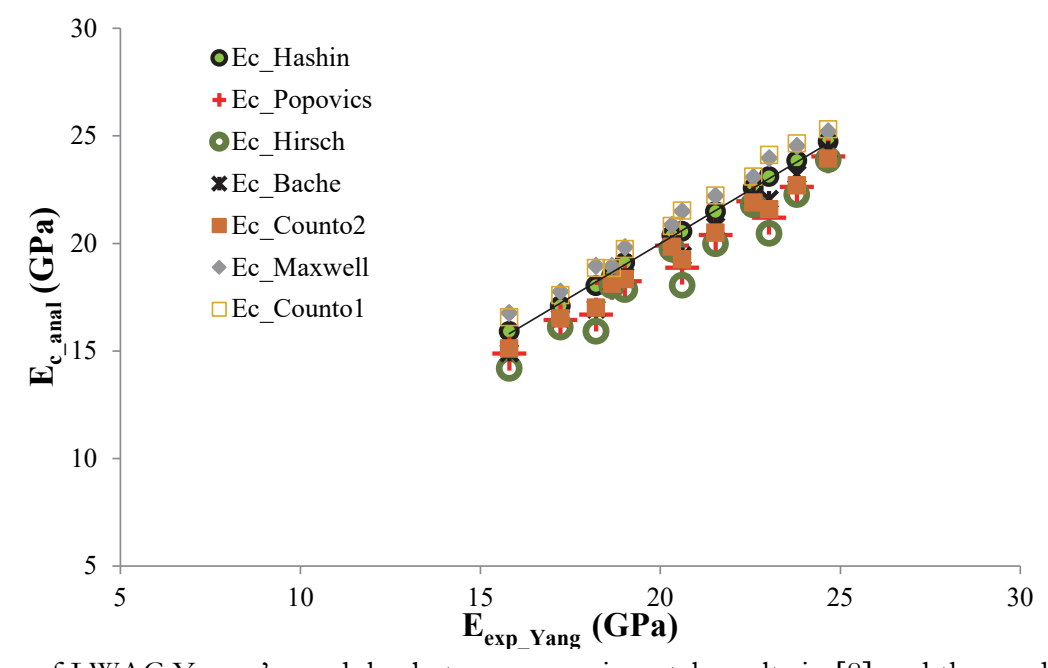

Figure 3: Confrontation of LWAC Young's modulus between experimental results in [8] and the predictions of 07 composite material models. 


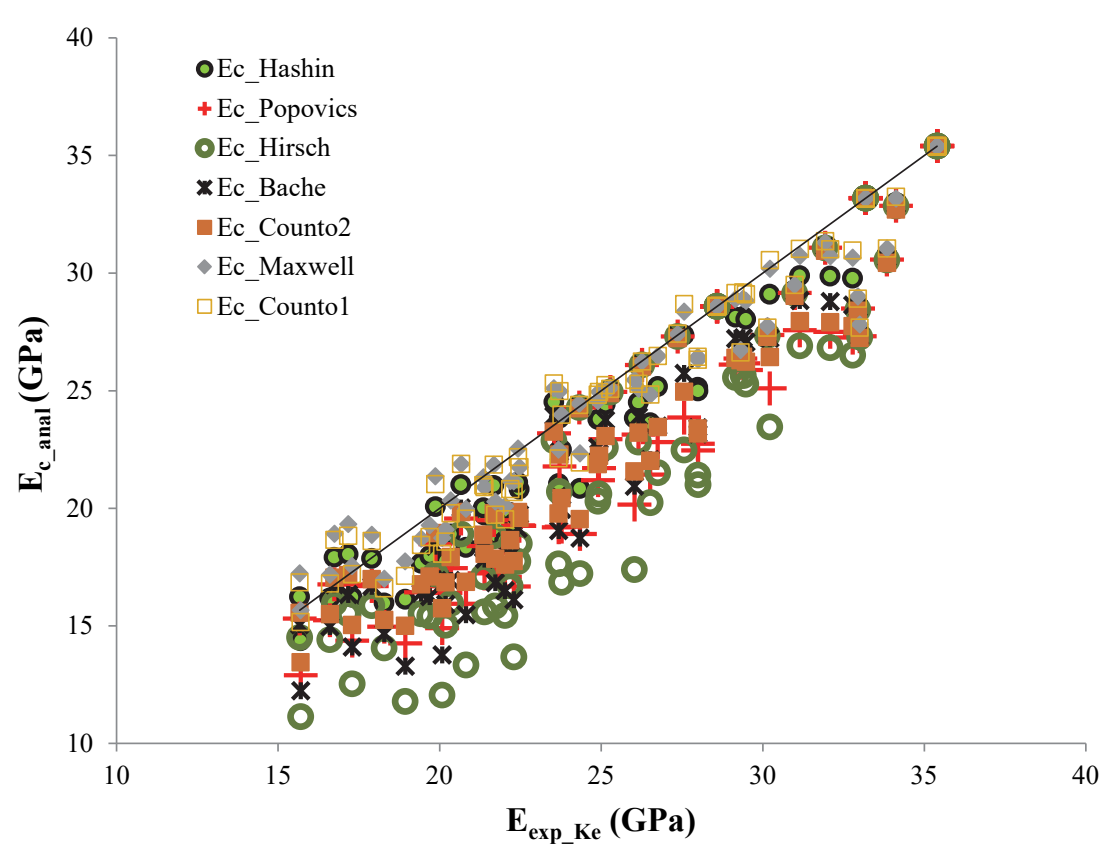

Figure 4: Confrontation of LWAC Young's modulus between experimental results in [9] and the predictions of 07 composite material models.

\begin{tabular}{|c|c|c|c|c|c|c|c|}
\hline $\begin{array}{l}\text { Ref. } \\
\text { grav. }\end{array}$ & Popovics & $\begin{array}{l}\text { Hirsch- } \\
\text { Dougill }\end{array}$ & $\begin{array}{l}\text { Hashin- } \\
\text { Hansen }\end{array}$ & Maxwell & Counto1 & Counto2 & $\begin{array}{c}\text { Bache and Nepper- } \\
\text { Christensen }\end{array}$ \\
\hline 8 Argi 16 & 0.68 & -1.62 & 4.48 & 8.87 & 7.42 & 1.21 & 0.46 \\
\hline 8 Isol S & 0.05 & -0.21 & 0.89 & 2.46 & 1.93 & -0.23 & 0.11 \\
\hline 8 Leca $7 j$. & 4.65 & 2.40 & 8.44 & 12.87 & 11.40 & 5.14 & 4.44 \\
\hline 8 Leca 28j. & -1.98 & -4.61 & 2.16 & 6.77 & 5.24 & -1.29 & -2.28 \\
\hline 8 Surex 675 & -0.09 & -0.15 & 0.18 & 0.93 & 0.67 & -0.33 & -0.06 \\
\hline 8 Galex 7j. & -1.21 & -1.28 & -1.60 & -0.69 & -1.03 & -2.13 & -1.30 \\
\hline Galex 28j. & 1.11 & 1.06 & 0.84 & 1.55 & 1.28 & 0.43 & 1.04 \\
\hline 9 Schiste $15 \mathrm{j}$. & -2.53 & -2.53 & -2.52 & -2.41 & -2.44 & -2.59 & -2.53 \\
\hline 9 Schiste $28 \mathrm{j}$. & 1.24 & 1.24 & 1.27 & 1.42 & 1.38 & 1.16 & 1.25 \\
\hline 9 Leca $1 j$. & -0.66 & -0.67 & -0.61 & -0.38 & -0.46 & -0.77 & -0.66 \\
\hline 9 Leca $2 j$. & -0.76 & -0.90 & -0.23 & 0.91 & 0.52 & -1.04 & -0.71 \\
\hline $9 \operatorname{Leca} 7 j$. & 1.42 & 0.70 & 3.12 & 5.68 & 4.82 & 1.27 & 1.45 \\
\hline 9 Leca $28 j$. & 1.05 & -0.76 & 4.27 & 8.20 & 6.90 & 1.36 & 0.92 \\
\hline 9 Leca 90j. & 2.93 & -0.63 & 8.07 & 13.50 & 11.71 & 3.98 & 2.42 \\
\hline 9 Surex $1 j$. & -5.36 & -5.48 & -5.96 & -4.79 & -5.22 & -6.64 & -5.53 \\
\hline 9 Surex $2 j$. & -3.25 & -3.26 & -3.30 & -3.08 & -3.15 & -3.44 & -3.26 \\
\hline 9 Surex $7 j$. & -4.95 & -4.95 & -4.95 & -4.94 & -4.95 & -4.96 & -4.95 \\
\hline 9 Surex 28j. & 0.52 & 0.52 & 0.60 & 0.91 & 0.81 & 0.39 & 0.54 \\
\hline 9 Surex $90 j$. & 11.96 & 11.87 & 12.37 & 13.39 & 13.04 & 11.67 & 12.00 \\
\hline LWC1 Crush & 0.66 & -0.21 & 2.66 & 5.43 & 4.56 & 0.57 & 0.74 \\
\hline 3 LWC1 Crush & -4.73 & -5.55 & -2.83 & -0.21 & -1.03 & -4.81 & -4.65 \\
\hline 3 LWC1 Pellet & 1.54 & 0.80 & 3.29 & 5.88 & 5.01 & 1.40 & 1.57 \\
\hline 3 LWC1 Pellet & -5.15 & -5.84 & -3.51 & -1.09 & -1.90 & -5.28 & -5.12 \\
\hline 3 HSLWC Pel. & 6.65 & 5.68 & 9.82 & 12.61 & 12.57 & 6.45 & 7.78 \\
\hline 3 HSLWC Pel. & 4.02 & 3.06 & 7.11 & 9.82 & 9.79 & 3.81 & 5.11 \\
\hline 1 Liapor & -0.03 & -0.05 & 0.07 & 0.49 & 0.30 & -0.17 & -0.04 \\
\hline 2 Liapor & 0.52 & 0.51 & 0.57 & 0.85 & 0.74 & 0.40 & 0.52 \\
\hline 16 Javron & 0.52 & 0.48 & 0.71 & 1.34 & 1.07 & 0.34 & 0.52 \\
\hline $16 \mathrm{G} / \mathrm{S}-0.2$ & 2.16 & 2.11 & 2.41 & 3.14 & 2.86 & 1.94 & 2.17 \\
\hline $16 \mathrm{G} / \mathrm{S}+0.2$ & -1.61 & -1.64 & -1.47 & -0.94 & -1.18 & -1.77 & -1.62 \\
\hline $16 \mathrm{EAU}+$ & 0.09 & -0.01 & 0.47 & 1.48 & 1.04 & -0.13 & 0.07 \\
\hline $16 \mathrm{EAU}-$ & 2.84 & 2.83 & 2.91 & 3.22 & 3.09 & 2.72 & 2.84 \\
\hline
\end{tabular}

Table 8: Error percentages of composite models and experimental results in [7] (\%). 
It can be observed from Tab. 8 that:

For the Hirsch-Dougill, Popovics, Bache and Nepper-Christensen, Counto2 and Hashin-Hansen models, 31/32 cases lead to $|\Delta \mathrm{E}|$ smaller than $10 \%$. All these models give a maximum $|\Delta \mathrm{E}|$ for a contrast equal to $\mathrm{E}_{\mathrm{g}} / \mathrm{E}_{\mathrm{m}}=61.29 \%$ with a volume fraction of the aggregates $\mathrm{Vg}=41.4 \%$ (aggregate: 9 Surex $90 \mathrm{j}$.).

For the Popovics and Bache and Nepper-Christensen models, $28 / 32$ cases give $|\Delta \mathrm{E}|$ smaller than $5 \%$ and $3 / 32$ cases give $|\Delta \mathrm{E}|$ smaller than $10 \%$. $\Delta \mathrm{E}$ ranges from $-5.53 \%$ to $12.00 \%$.

For the Hirsch-Dougill and Counto 2 models, $27 / 32$ cases give $|\Delta \mathrm{E}|$ smaller than $5 \%$ and $4 / 32$ cases give $|\Delta \mathrm{E}|$ smaller than $10 \%$. $\Delta$ E ranges from $-5.84 \%$ to $11.87 \%$.

For the Hashin-Hansen model, $31 / 32$ cases lead to $|\Delta \mathrm{E}|$ smaller than $10 \%$. Hence, for $26 / 32$ cases it is smaller than $5 \%$. $\Delta \mathrm{E}$ ranges from $-5.96 \%$ to $12.37 \%$.

For the Counto1 and Maxwell models, $28 / 32$ cases lead to $|\Delta \mathrm{E}|$ smaller than $10 \%, \Delta \mathrm{E}$ ranges from $-4.94 \%$ to $13.50 \%$.

It is clear from these results that the selected models are able to effectively estimate the Young's modulus of LWAC tested by De Larrard [7] with a max difference $|\Delta \mathrm{E}|$ equal to $13.50 \%$ (obtained by the Maxwell model) using 32 measurements.

\begin{tabular}{|c|c|c|c|c|c|c|c|}
\hline $\begin{array}{l}\text { Ref. } \\
\text { grav. }\end{array}$ & Popovics & $\begin{array}{l}\text { Hirsch- } \\
\text { Dougill }\end{array}$ & $\begin{array}{l}\text { Hashin- } \\
\text { Hansen }\end{array}$ & Maxwell & Counto1 & Counto2 & $\begin{array}{l}\text { Bache and } \\
\text { Nepper- } \\
\text { Christensen }\end{array}$ \\
\hline A3 & -7.90 & -11.07 & 0.36 & 4.11 & 4.78 & -6.22 & -4.22 \\
\hline A4 & -8.36 & -12.35 & -0.20 & 4.37 & 4.48 & -6.48 & -5.72 \\
\hline A5 & -8.29 & -12.57 & -0.94 & 4.13 & 3.57 & -6.54 & -6.90 \\
\hline A6 & -5.83 & -10.19 & 0.66 & 6.14 & 4.87 & -4.28 & -5.60 \\
\hline B3 & -4.85 & -6.36 & 0.25 & 3.11 & 3.63 & -4.54 & -2.49 \\
\hline B4 & -5.26 & -7.17 & -0.23 & 3.20 & 3.28 & -4.77 & -3.47 \\
\hline B5 & -4.01 & -6.04 & 0.50 & 4.26 & 3.84 & -3.53 & -2.94 \\
\hline B6 & -4.50 & -6.44 & -0.72 & 3.10 & 2.21 & -4.09 & -4.12 \\
\hline C3 & -2.49 & -3.09 & 0.26 & 2.25 & 2.62 & -2.90 & -1.17 \\
\hline $\mathrm{C} 4$ & -2.73 & -3.48 & -0.05 & 2.27 & 2.33 & -3.01 & -1.70 \\
\hline C5 & -2.07 & -2.82 & 0.24 & 2.69 & 2.41 & -2.29 & -1.42 \\
\hline C6 & -2.59 & -3.28 & -0.74 & 1.66 & 1.09 & -2.79 & -2.30 \\
\hline
\end{tabular}

Table 9: Error percentages of composite models and experimental results in [8] (\%).

Compared with the experimental data of Yang and Huang [8] (Tab. 6, Tab. 9), Bache and Nepper-Christensen, Counto2, Popovics, Hirsch-Dougill, underestimate the measured Young's modulus. On the other hand, the Maxwell and Counto1 models overestimate the Young's modulus measured by Yang and Huang [8].

As seen in Tab. 9, for the Hashin-Hansen and Counto1 models, $12 / 12$ cases give $|\Delta \mathrm{E}|$ smaller than $5 \% .|\Delta \mathrm{E}|$ ranges from $0.94 \%$ to $4.87 \%$. The Maxwell gives $12 / 12$ cases smaller than $10 \%$ and $11 / 12$ smaller than $5 \%$. $|\Delta \mathrm{E}|$ ranges from $1.66 \%$ to $6.14 \%$. In all composite models, the error percentages differ between $0.05 \%$ and $12.57 \%$.

It can be seen that the most accurate models are those of Hashin-Hansen, Counto1 and Maxwell which give less errors percentages.

The predictions of the LWAC Young's modulus using the 07 composite material models are compared with experimental data of Ke Y et al. [9] (Tab. 7 and Tab.10) in Fig. 4. All selected composite models appear applicable to predict the Young's modulus of LWAC tested by Ke Y et al [9].

For the Maxwell model, $50 / 75$ cases give $|\Delta \mathrm{E}|$ smaller than $5 \%$ and $22 / 75$ cases smaller than $10 \%$. This means that $72 / 75$ cases have $|\Delta \mathrm{E}|$ smaller than $10 \%$. This model converges on the experimental values measured by Ke $\mathrm{Y}$ et al. [9] with an absolute maximum difference $|\Delta \mathrm{E}|$ of $15.72 \%$.

For the Counto1 model, $47 / 75$ cases lead to $|\Delta \mathrm{E}|$ smaller than $5 \%$ and $23 / 75$ smaller than $10 \%$, which gives $70 / 75$ cases with $|\Delta \mathrm{E}|$ smaller than $10 \%$, with a maximum difference of $16.14 \%$.

For the Hashin-Hansen model, $59 / 75$ cases give $|\Delta \mathrm{E}|$ smaller than $10 \%$ of which $38 / 75$ cases smaller than $5 \%$. $\Delta \mathrm{E}$ ranges from $0 \%$ to $16.77 \%$.

For the Counto 2 model, $36 / 75$ cases have $|\Delta \mathrm{E}|$ smaller than $10 \%$, of which 27 cases have $|\Delta \mathrm{E}|$ smaller than $5 \%$, with the maximum difference of $21.59 \%$.

For the Popovics model, $34 / 75$ cases give $|\Delta \mathrm{E}|$ smaller than $10 \%$ with $25 / 75$ cases smaller than $5 \%$. The maximum $\Delta \mathrm{E}$ is $25.78 \%$. 


\begin{tabular}{|c|c|c|c|c|c|c|c|}
\hline $\begin{array}{l}\text { Ref. } \\
\text { grav. }\end{array}$ & Popovics & $\begin{array}{l}\text { Hirsch- } \\
\text { Dougill }\end{array}$ & $\begin{array}{l}\text { Hashin- } \\
\text { Hansen }\end{array}$ & Maxwell & Counto1 & Counto2 & $\begin{array}{l}\text { Bache and } \\
\text { Nepper- } \\
\text { Christensen }\end{array}$ \\
\hline \multirow{5}{*}{$\begin{array}{l}\text { M8 } \\
0 / 4650 \text { A }\end{array}$} & 0.00 & 0.00 & 0.00 & 0.00 & 0.00 & 0.00 & 0.00 \\
\hline & 4.18 & -2.84 & 1.52 & 6.63 & 7.49 & 1.21 & 1.62 \\
\hline & 1.60 & -8.53 & 5.33 & 5.91 & 5.91 & 4.03 & -3.14 \\
\hline & 6.96 & -4.71 & 0.12 & 12.96 & 11.40 & 1.80 & 0.03 \\
\hline & 3.61 & -7.43 & 2.31 & 9.98 & 7.50 & 0.72 & -3.95 \\
\hline \multirow{5}{*}{$\begin{array}{l}\text { M8 } \\
4 / 10550 \mathrm{~A}\end{array}$} & 0.00 & 0.00 & 0.00 & 0.00 & 0.00 & 0.00 & 0.00 \\
\hline & 6.34 & -12.80 & 11.56 & 4.10 & 3.32 & 11.25 & -8.68 \\
\hline & 3.34 & -13.19 & 10.06 & 0.81 & 0.81 & 8.76 & -7.95 \\
\hline & 0.24 & -11.38 & 6.74 & 5.42 & 3.95 & 5.12 & -6.84 \\
\hline & 2.59 & -13.21 & 8.25 & 3.49 & 1.13 & 6.71 & -9.85 \\
\hline \multirow{5}{*}{$\begin{array}{l}\text { M8 } \\
4 / 10430 \mathrm{~A}\end{array}$} & 0.00 & 0.00 & 0.00 & 0.00 & 0.00 & 0.00 & 0.00 \\
\hline & 4.54 & -18.49 & 14.88 & 1.36 & 0.29 & 12.14 & -9.29 \\
\hline & 7.91 & -27.09 & 19.47 & 2.32 & 2.32 & 15.56 & -16.58 \\
\hline & 6.23 & -27.52 & 16.93 & 1.45 & 0.52 & 13.02 & -18.47 \\
\hline & 8.55 & -29.03 & 17.79 & 0.20 & 3.42 & 14.33 & -22.04 \\
\hline \multirow{5}{*}{$\begin{array}{l}\text { M8 } \\
4 / 10520 \mathrm{~S}\end{array}$} & 0.00 & 0.00 & 0.00 & 0.00 & 0.00 & 0.00 & 0.00 \\
\hline & 2.87 & -10.18 & 8.74 & 0.45 & 0.39 & 8.23 & -5.50 \\
\hline & 7.40 & -17.68 & 14.32 & 3.25 & 3.25 & 12.85 & -12.18 \\
\hline & 9.09 & -20.14 & 15.44 & 3.69 & 5.09 & 13.75 & -15.61 \\
\hline & 12.72 & -23.09 & 18.15 & 7.02 & 9.24 & 16.60 & -19.78 \\
\hline \multirow{5}{*}{$\begin{array}{l}\text { M8 } \\
4 / 10750 \mathrm{~S}\end{array}$} & 0.00 & 0.00 & 0.00 & 0.00 & 0.00 & 0.00 & 0.00 \\
\hline & 0.12 & -0.23 & 0.23 & 0.11 & 0.20 & 0.48 & -0.16 \\
\hline & 0.48 & -0.65 & 0.63 & 0.09 & 0.09 & 0.90 & -0.57 \\
\hline & 1.16 & -1.34 & 1.32 & 0.68 & 0.81 & 1.53 & -1.28 \\
\hline & 0.01 & -0.18 & 0.16 & 0.51 & 0.30 & 0.33 & -0.15 \\
\hline \multirow{5}{*}{$\begin{array}{l}\text { M9 } \\
0 / 4650 \mathrm{~A}\end{array}$} & 0.00 & 0.00 & 0.00 & 0.00 & 0.00 & 0.00 & 0.00 \\
\hline & 4.25 & -12.79 & 10.99 & 1.67 & 0.78 & 10.07 & -7.29 \\
\hline & 0.46 & -12.67 & 8.16 & 5.34 & 5.34 & 6.03 & -5.60 \\
\hline & 0.98 & -13.46 & 7.06 & 7.51 & 5.82 & 4.70 & -7.49 \\
\hline & 5.04 & -9.68 & 2.40 & 12.54 & 9.63 & 0.09 & -4.89 \\
\hline \multirow{5}{*}{$\begin{array}{l}\text { M9 } \\
4 / 10550 \mathrm{~A}\end{array}$} & 0.00 & 0.00 & 0.00 & 0.00 & 0.00 & 0.00 & 0.00 \\
\hline & 3.55 & -12.34 & 10.46 & 0.93 & 0.03 & 9.49 & -6.67 \\
\hline & 4.63 & -17.34 & 12.94 & 0.05 & 0.05 & 10.85 & -10.49 \\
\hline & 6.30 & -19.97 & 13.88 & 0.18 & 1.76 & 11.63 & -14.30 \\
\hline & 8.71 & -21.76 & 15.27 & 2.12 & 4.67 & 13.21 & -17.50 \\
\hline \multirow{5}{*}{$\begin{array}{l}\text { M9 } \\
4 / 10430 \mathrm{~A}\end{array}$} & 0.00 & 0.00 & 0.00 & 0.00 & 0.00 & 0.00 & 0.00 \\
\hline & 0.74 & -18.47 & 13.48 & 2.85 & 4.05 & 9.48 & -6.66 \\
\hline & 5.44 & -29.14 & 19.17 & 0.81 & 0.81 & 14.08 & -16.08 \\
\hline & 11.94 & -35.93 & 23.46 & 4.05 & 6.07 & 18.96 & -25.67 \\
\hline & 14.83 & -37.78 & 24.69 & 6.29 & 9.58 & 20.77 & -29.84 \\
\hline \multirow{5}{*}{$\begin{array}{l}\text { M9 } \\
4 / 10520 \mathrm{~S}\end{array}$} & 0.00 & 0.00 & 0.00 & 0.00 & 0.00 & 0.00 & 0.00 \\
\hline & 4.90 & -14.27 & 12.21 & 2.23 & 1.31 & 11.02 & -8.21 \\
\hline & 10.91 & -23.71 & 19.18 & 6.38 & 6.38 & 16.97 & -16.79 \\
\hline & 10.71 & -24.74 & 18.37 & 4.64 & 6.21 & 16.00 & -18.90 \\
\hline & 11.97 & -25.54 & 18.68 & 5.36 & 7.92 & 16.50 & -21.07 \\
\hline \multirow{5}{*}{$\begin{array}{l}\text { M9 } \\
4 / 10750 \mathrm{~S}\end{array}$} & 0.00 & 0.00 & 0.00 & 0.00 & 0.00 & 0.00 & 0.00 \\
\hline & 2.38 & -2.70 & 2.68 & 1.97 & 1.80 & 3.09 & -2.51 \\
\hline & 5.52 & -5.98 & 5.93 & 4.82 & 4.82 & 6.31 & -5.76 \\
\hline & 8.81 & -9.29 & 9.21 & 7.95 & 8.18 & 9.48 & -9.13 \\
\hline & 9.71 & -10.15 & 10.06 & 8.80 & 9.16 & 10.28 & -10.06 \\
\hline \multirow{5}{*}{$\begin{array}{l}\text { M10 } \\
0 / 4650 \mathrm{~A}\end{array}$} & 0.00 & 0.00 & 0.00 & 0.00 & 0.00 & 0.00 & 0.00 \\
\hline & 4.04 & -13.61 & 11.50 & 1.32 & 0.39 & 10.26 & -7.41 \\
\hline & 5.89 & -19.58 & 14.71 & 1.07 & 1.07 & 12.34 & -12.18 \\
\hline & 5.92 & -20.89 & 14.07 & 0.51 & 1.15 & 11.54 & 14.65 \\
\hline & 7.08 & -21.59 & 14.24 & 0.06 & 2.78 & 11.90 & -16.81 \\
\hline
\end{tabular}




\begin{tabular}{|c|c|c|c|c|c|c|c|}
\hline \multirow{5}{*}{$\begin{array}{l}\text { M10 } \\
4 / 10550 \mathrm{~A}\end{array}$} & 0.00 & 0.00 & 0.00 & 0.00 & 0.00 & 0.00 & 0.00 \\
\hline & 6.93 & -16.40 & 14.29 & 4.27 & 3.36 & 13.03 & -10.26 \\
\hline & 10.20 & -23.51 & 18.75 & 5.56 & 5.56 & 16.42 & -16.31 \\
\hline & 11.14 & -25.55 & 18.95 & 5.01 & 6.59 & 16.50 & -19.54 \\
\hline & 13.25 & -27.05 & 20.03 & 6.63 & 9.19 & 17.80 & -22.49 \\
\hline \multirow{5}{*}{$\begin{array}{l}\text { M10 } \\
4 / 10430 \text { A }\end{array}$} & 0.00 & 0.00 & 0.00 & 0.00 & 0.00 & 0.00 & 0.00 \\
\hline & 3.72 & -22.37 & 16.94 & 0.13 & 1.06 & 12.55 & -9.90 \\
\hline & 8.48 & -33.18 & 22.55 & 2.24 & 2.24 & 17.16 & -19.54 \\
\hline & 13.21 & -38.63 & 25.19 & 5.16 & 7.22 & 20.40 & -27.73 \\
\hline & 15.46 & -39.97 & 25.78 & 6.67 & 10.05 & 21.59 & -31.45 \\
\hline \multirow{5}{*}{$\begin{array}{l}\text { M10 } \\
4 / 10520 \mathrm{~S}\end{array}$} & 0.00 & 0.00 & 0.00 & 0.00 & 0.00 & 0.00 & 0.00 \\
\hline & 9.18 & -19.13 & 16.85 & 6.49 & 5.58 & 15.37 & -12.66 \\
\hline & 10.78 & -24.97 & 19.78 & 6.01 & 6.01 & 17.20 & -17.27 \\
\hline & 14.37 & -29.26 & 22.32 & 8.24 & 9.82 & 19.74 & -23.02 \\
\hline & 15.45 & -29.90 & 22.42 & 8.74 & 11.34 & 20.06 & -25.09 \\
\hline \multirow{5}{*}{$\begin{array}{l}\text { M10 } \\
4 / 10750 \mathrm{~S}\end{array}$} & 0.00 & 0.00 & 0.00 & 0.00 & 0.00 & 0.00 & 0.00 \\
\hline & 3.29 & -3.74 & 3.71 & 2.77 & 2.57 & 4.18 & -3.47 \\
\hline & 9.11 & -9.75 & 9.66 & 8.27 & 8.27 & 10.07 & -9.44 \\
\hline & 12.99 & -13.65 & 13.52 & 11.97 & 12.24 & 13.80 & -13.43 \\
\hline & 16.77 & -17.36 & 17.22 & 15.72 & 16.14 & 17.43 & -17.23 \\
\hline
\end{tabular}

Table 10: Error percentages of composite models and experimental results in [9] (\%).

The Bache and Nepper-Christensen and Hirsch-Dougill models underestimate the Young's modulus of LWAC measured in [3]. Bache and Nepper-Christensen model, $43 / 75$ cases give $|\Delta \mathrm{E}|$ smaller than $10 \%$ and $\Delta \mathrm{E}$ ranges from $31.45 \%$ to $1.62 \%$.

For the Hirsch-Dougill model, $29 / 75$ cases give $|\Delta \mathrm{E}|$ smaller than $10 \%$ with 23 cases smaller than $5 \%$.

It can be seen by examining Fig. 4 that the most accurate models are those of Maxwell, Counto1 and Hashin-Hansen which give less errors percentages (Fig. 4 and Tab. 10).

\section{Statistical analysis}

In order to confirm what has been announced previously and distinguish the most suitable model for predicting the effective elasticity modulus of the LWAC, a global statistical study was carried out on all the experimental values of the three researchers (119 measures). To this effect, the mean values and standard deviation for all composite models used in this study and experimental data are calculated as seen in Tab. 10.

\begin{tabular}{cccccccc}
\hline & Popovics & $\begin{array}{l}\text { Hirsch- } \\
\text { Dougill }\end{array}$ & $\begin{array}{c}\text { Hashin- } \\
\text { Hansen }\end{array}$ & Maxwell & Counto1 & Counto2 & $\begin{array}{c}\text { Bache and } \\
\text { Nepper- } \\
\text { Christensen }\end{array}$ \\
Mean Values & -6.90 & -9.66 & -2.72 & 0.29 & -0.23 & -5.94 & -6.42 \\
Standard deviation & 8.24 & 11.14 & 5.72 & 5.27 & 5.32 & 7.12 & 8.46 \\
\hline
\end{tabular}

Table 10: Mean values and standard deviation of composite models and all experimental data in [7, 8, 9].

Fig. 5 shows the normal distribution approximation of error percentage for all 07 composite analytical models. Every estimator has a pick on the mean value and a standard deviation presented by a tight or wide curve. As expected, the Maxwell, Counto1 and Hashin-Hansen composite models provide a good prediction of experimental Young's modulus of all LWAC tested by De Larrard [7], Yang and Huang [8] and Ke Y et al. [9] (119 values) with a maximum volume fraction of aggregates $\mathrm{Vg}$ equal to $49.37 \%$.

It is clear from curves of Fig. 5 that the best curves that fit experimental data are respectively Maxwell, Counto1 and Hashin-Hansen models because the mean values are closest to zero than others. It is also important to notice that the standard deviation of both models (Maxwell 5.27, Counto1 5.32 and Hashin-Hansen 5.72) are tight which indicates that there is a high concentration of estimated values around of zero. 

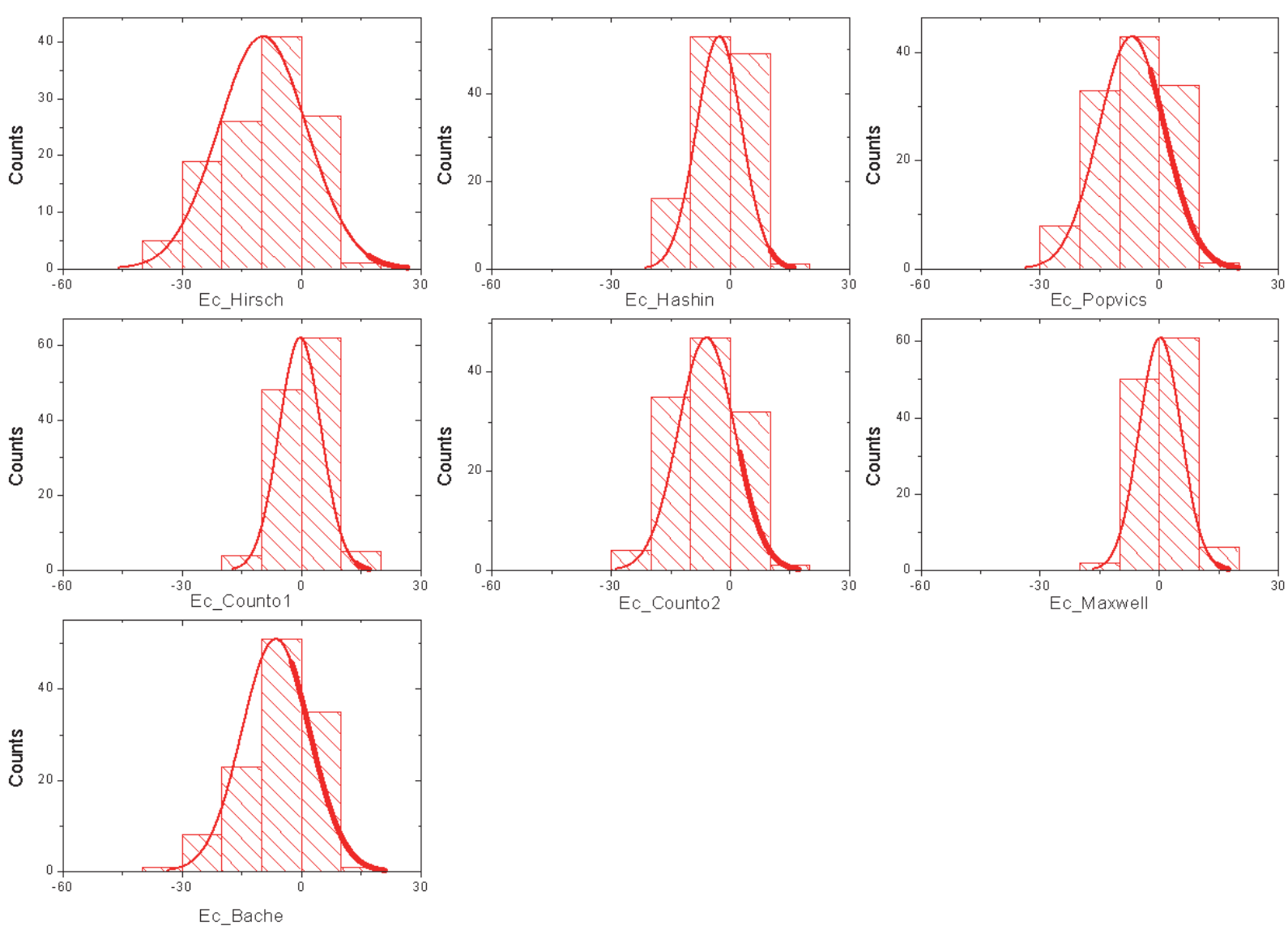

Figure 5: Error percentage distribution approximation to normal statistic low of each composite material model.

\section{CONCLUSION}

7 he modulus of elasticity is a very important mechanical parameter, its determination sometimes involves impossible, difficult or costly tests, the alternative use of the biphasic laws in these cases appears very interesting but the choice of a model and not another remains a question which requires a precise examination and strongly depends on the type of materials chosen.

In order to choose the optimized prediction composite model for Lightweight Aggregate Concrete, the purpose of this paper was to appraise the effective Young's modulus of LWAC using two-phase composite models. From the obtained numerical predictions, as confronted to existing experimental data and analytical results, the main findings are summarized below:

When the Young's modulus of lightweight aggregates $\mathrm{E}_{\mathrm{g}}$ is much less than the Young's modulus of the mortar matrix in the lightweight aggregate concrete $\mathrm{E}_{\mathrm{m}}$, Hirsch-Dougill models remain distant from experimental results and cannot be applied to predict the modulus of elasticity of LWAC.

Using Popovics, Counto2 and Bache-Nepper Christensen composite models may not always produce accurate results.

For 119 experimental values of Young's modulus for LWAC, the Maxwell, Counto1 and Hashin-Hansen seem the most reasonable for this purpose.

The Maxwell model takes into account in the calculation of the effective elastic modulus of the contrast between the two phases (the mortar matrix and the light aggregates) represented by the coefficient $\alpha\left(E_{g} / E_{m}\right)$ which made it possible to simulate the materials well and offered consequently more precise results if compared with other models. Thus, the precision of this prediction model demonstrates its effectiveness and potential application as a model for Lightweight Aggregate Concrete. The Maxwell model remains close from the experimental values with a man value error equal to 0.29 and a standard deviation equal to 5.27. In addition the Counto1 and Hashin-Hansen models provide a good prediction of 
experimental Young's modulus of all LWAC tested by De Larrard [7], Yang and Huang [8] and Ke Y et al. [9] (119 values) with a maximum volume fraction of aggregates $\mathrm{Vg}$ equal to $49.37 \%$.

In conclusion, it can be suggested that additional studies about investigation for predicting modulus of elasticity of LWAC, may contribute to confirm the reliability and the accuracy of Maxwell, Counto1 and Hashin-Hansen models.

\section{NOMENCLATURE}

E: Young's modulus

$\mathrm{E}_{\mathrm{g}}$ : Young's modulus of lightweight aggregate (dispersed phase)

$\mathrm{E}_{\mathrm{m}}$ : Young's modulus of matrix (mortar)

$\mathrm{V}_{\mathrm{g}}$ : Volume fraction of aggregate (dispersed phase)

$\mathrm{V}_{\mathrm{m}}: \quad$ Volume fraction of matrix (mortar)

$\mathrm{E}_{\mathrm{c}}$ : Young's modulus of composite

$\mathrm{E}_{\mathrm{c}_{-} \text {Voigt }}$ : Young's modulus of composite using Voigt model (upper bound)

$\mathrm{E}_{\mathrm{c} \_ \text {Reuss }}$ : Young's modulus of composite using Reuss model (lower bound)

$\mathrm{E}_{\mathrm{c}_{-} \text {Hashin }}$ : Young's modulus of composite using Hashin-Hansen model

$\mathrm{E}_{\mathrm{c} \_ \text {Hirsch }}$ : Young's modulus of composite using Hirsch-Dougill model

$\mathrm{E}_{\mathrm{c}_{-} \text {Popovics }}$ : Young's modulus of composite using Popovics model

$\mathrm{E}_{\mathrm{c} \_ \text {Maxwell }}$ : Young's modulus of composite using Maxwell model

$\alpha$ : Empirical factor

$\mathrm{E}_{\mathrm{c}_{-} \text {Countol }}$ : Young's modulus of composite using Counto1 model

$\mathrm{E}_{\mathrm{c} \_ \text {Counto2 }}$ : Young's modulus composite using Counto2 model

$\mathrm{E}_{\mathrm{c}_{-} \text {Bache }}$ : Young's modus of composite using Bache and Nepper-Christensen model

$\mathrm{E}_{\exp _{\mathrm{De}} \text { Larrard }}$ : Young's modulus of LWAC tested by De Larrard and Le Roy (1995)

$\mathrm{E}_{\text {exp_Yang }}$ : Young's modulus of LWAC tested by Yang and Huang (1998)

$\mathrm{E}_{\text {exp_Ke }}$ : Young's modulus of LWAC tested by Ke Y et al (2010)

$v_{\mathrm{c}}: \quad$ Poisson's ratio of composite

$v_{\mathrm{m}}: \quad$ Poisson's ratio of matrix (mortar)

$v_{\mathrm{g}}$ : Poisson's ratio of aggregate (dispersed phase)

$\mathrm{d}$ : Smallest diameter of aggregates in concrete

D: Largest diameter of aggregates in concrete

$\mathrm{E}_{\mathrm{c} \_ \text {anal }}$ : Young's modulus predicted from analytic model

$\Delta \mathrm{E}:$ Error percentage

$|\Delta \mathrm{E}|:$ Absolute value of error percentage

\section{REFERENCES}

[1] Muhammad Riaz, A. and Bing, C. (2019). Experimental research on the performance of lightweight concrete containing foam and expanded clay aggregate, Composites Part B: Engineering, 171, pp. 46-60.

DOI: $10.1016 /$ j.compositesb.2019.04.25 
[2] Rashad, A. M. (2018). Lightweight expanded clay aggregate as a building material - An overview. Construction and Building Materials, 170, pp. 757-775. DOI: 10.1016/j.conbuildmat.2018.03.009.

[3] Colangelo, F. and Ilenia, F. (2019). Lightweight concrete with polyolefins as aggregates. Use of Recycled Plastics in Eco-efficient Concrete, pp. 167-187, Elsevier. DOI: 10.1016/B978-0-08-102676-2.00008-6.

[4] Muhammad Riaz, A., Bing, C. and Farasat Ali Shah, S. (2019). Investigate the influence of expanded clay aggregate and silica fume on the properties of lightweight concrete, Construction and Building Materials, 220, pp. 253-266. DOI: $10.1016 /$ j.conbuildmat.2019.05.171

[5] Chandra, S., Berntsson, L. (2002). Lightweight Aggregate Concrete. Elsevier.

[6] Ke, Y., Beaucour, A. L., Ortola, S., Dumontet, H., Cabrillac, R. (2009). Influence of volume fraction and characteristics of lightweight aggregates on the mechanical properties of concrete, Constr. Build. Mater. 23(8), pp. 2821-2828. DOI: 10.1016/j.conbuildmat.2009.02.038

[7] De Larrard, F. (1995). Une Approche de la Formulation des Bétons Légers de Structure, Bulletin de liaison des laboratoires des Ponts et Chaussées LCPC, 195, pp. 39-47 (in French).

[8] Yang, C. C., Huang, R. (1998). Approximation Strength of Lightweight Aggregate Using Micromechanics Method, Adv. Cem. Based Mater, 7(3-4), pp. 113-138. DOI: 10.1016/S1065-7355(98)00002-9.

[9] Ke, Y., Ortola, S., Beaucour, A.L., Dumontet, H. (2010). Identification of Microstructural Characteristics in Lightweight Aggregate Concretes by Micromechanical Modelling Including the Interfacial Transition Zone (ITZ). Cem. Conc. Res., 40(11), pp. 1590-1600. DOI: 10.1016/j.cemconres.2010.07.001.

[10] Kurugöl, S., Tanaçan, L., Ersoy, H.S. (2008). Young's Modulus of Fiber-Reinforced and Polymer-Modified Lightweight Concrete Composites, Constr. Build. Mater, 22(6), pp. 1019-1028.

DOI: $10.1016 /$ j.conbuildmat.2007.03.017.

[11] Tanaçan, L. Ersoy, H.Y. (2000). Mechanical Properties of Fired Clay-Perlite as Composite Material, J. Mater. Civil Eng. ASCE, 12(1), pp. 55-59. DOI: 10.1061/(ASCE)0899-1561(2000)12:1(55).

[12] Cui, HZ. (2011). Study of prediction model for compressive strength of lightweight aggregate concrete. Adv Mater Res, pp. 1204-1209. DOI: 10.4028/www.scientific.net/AMR.335-336.1204

[13] Lo, T.Y., Cui, H.Z. (2004). Effect of porous lightweight aggregate on strength of concrete, Mater. Lett. 58, pp. 916919. DOI: 10.1016/j.matlet.2003.07.036.

[14] Ardakani, A., Yazdani, M. (2014). The relation between particle density and static-elastic moduli of lightweight expanded clay aggregates, Appl. Clay Sci. 93-94, pp. 28-34. DOI: 10.1016/j.clay.2014.02.017.

[15] Ohama, Y. (1987). Principle of Latex Modification and Some Typical Properties of Latex-Modified Mortar and Concrete, ACI Mater. J., 84(6), pp. 511-518.

[16] Kocataskin, F., Özturan, T., Ersoy, H.Y. (1988). A Composite Materials Approach For the Prediction of Concrete Properties, Bull. Techn. Univer. Istanbul, 41(2), pp. 333-347.

[17] Zhou, F.P., Lydon, F.D., Barr, B.I.G. (1995). Effect of Coarse Aggregate on Elastic Modulus and Compressive Strength of High Performance Concrete, Cem. Conc. Res., 25(1), pp. 177-186. DOI: 10.1016/0008-8846(94)00125-I.

[18] Gilormini, P., Bréchet, Y. (1999). Syntheses: Mechanical Properties of Heterogeneous Media: Which Material For Which Model? Which Model For Which Material?, Mod. Simul. Mater. Sci. Eng., 7(5), pp. 805-816. DOI: $10.1088 / 0965-0393 / 7 / 5 / 312$.

[19] Hansen, T.C. (1960). Strength Elasticity and creep as related to the internal structure of concrete. In: Chemistry of cement, Proceedings of fourth international symposium, Monograph, 2, pp. 709-723, Washington.

[20] Popovics, S., Erdey, M. (1970). Estimation of the Modulus of Elasticity of Concrete-Like Composite Materials, M.R.A. Mater. Struct., 3(4), pp. 253-260. DOI: 10.1007/BF02474013.

[21] Hirsch, T.J. (1962). Modulus of elasticity of concrete affected by elastic moduli of cement paste matrix and aggregate, ACI J., 59(3), pp. 427-452.

[22] Hansen, T.C. (1965). Influence of aggregate and voids on modulus of elasticity of concrete, cement mortar, and cement paste, ACI J., 62(2), pp. 193-216.

[23] Counto, U.J. (1964). The Effect of the Elastic Modulus of the Aggregate on the Elastic Modulus, Creep and Creep Recovery of Concrete, Mag. Conc. Res., 16(48), pp.129-138. DOI: 10.1680/macr.1964.16.48.129

[24] Bache, H.H., Nepper-Christansen, P. (1965). Observations on strength and fracture in lightweight and ordinary concrete-the Structure of concrete and its behavior under load, Proceedings of International Conference, Cement and Concrete Association, pp. 93-108, London.

[25] Nielsen, L.E., Chen, P.E. (1968). Young’s Modulus of Composites Filled With Randomly Oriented Short Fibers, J. Mat., 3(2), pp. 352-358.

[26] Hashin, Z. (1962). The elastic moduli of heterogeneous materials. J. Appl. Mech, 29(143). 